\title{
Analysis on Semiconductor Thermoelectric Cooler Temperature Control System Based on MCU
}

\author{
Juan Zhua* and Jianping Huang \\ Hitachi Air-Conditioning \& Refrigerating Products (Guangzhou) Co., Ltd., Guangzhou, Guangdong 510935, China
}

\begin{abstract}
With the progress of science and technology, material application science has developed rapidly. As a kind of new material, the semiconductor refrigeration material has been well applied. Based on that, this essay studies semiconductor thermoelectric cooler temperature control system based on MCU and its application. The research indicates that by controlling power supply's unit circuit with power dissipation, the system controls the working condition (refrigeration / heating switch, adjustable power) of semiconductor thermoelectric cooler to achieve the goal of measuring and controlling the temperature of some equipment, guaranteeing their proper functioning. The system is easy to operate, and it works safely and reliably, which could meet the overall performance requirements, and is of great application prospect and promotion value.
\end{abstract}

\author{
KEYWORDS \\ $\mathrm{MCU}$ \\ Semiconductor \\ Thermoelectric cooler \\ Temperature control
}

\section{Introduction}

Current refrigeration equipment mostly adopt compressor refrigeration technology, which has certain limitations, such as hard starting during low temperature, low power density, high installment requirement, high maintenance cost and so on. Semiconductor refrigeration technology adopts power electric circuit and digit controller technology, with advantages of no refrigerant, few mechanical components, small volume, high reliability, high control precision, little electromagnetic interference and so on.

Compared with traditional resource technology, semiconductor resource technology has significant differences, in particular its resource system working principle is essentially different from traditional refrigeration process. Due to its characteristics of no noise in the process of refrigeration, small dimension of refrigeration device,

Copyright $\odot 2015$ Juan Zhu and Jianping Huang

doi: 10.18686/esta.v2i1.1

Received: October 5, 2015; Accepted: November 8, 2015; Published online: December 3

This is an open-access article distributed under the terms of the Creative Commons Attribution Unported License (http://creativecommons.org/ licenses/by-nc/4.0/), which permits unrestricted use, distribution, and reproduction in any medium, provided the original work is properly cited.

${ }^{*}$ Corresponding author: Hitachi Air-Conditioning \& Refrigerating Products (Guangzhou)., Ltd., Guangzhou, Guangdong 510935, China. E-mail: zhujuan156@sina.com flexible arrangement and so on, semiconductor refrigeration technology has been widely applied in recent years, relevant study and application has been more and more deeper, and its coverage wider and wider. With the characteristic and advantage of semiconductor refrigeration technology, applying semiconductor refrigeration technology to design and realize the goal of controlling the temperature of small calorstat is a great solution. In the application process of realizing, relatively good application effect has been achieved, and previously relevant scholars' research also reflected that. However, with the constant expansion of application scenarios, the requirements for temperature control precision of calorstat based on semiconductor refrigeration technology has been higher and higher. In order to design high precision calorstat based on semiconductor technology, deeper research and analysis on semiconductor refrigeration principle are required, more precise semiconductor refrigeration working model is need to be established to design high precision semiconductor refrigeration calorstat, meeting users' requirements. This essay would design a high precision refrigeration algorithm based on semiconductor refrigeration technology by deeply analyzing and modeling semiconductor refrigeration process and working principle to meet the application requirements of high precision calorstat [1].

\section{Introduction of MCU}

The abbreviation of unit microcomputer is MCU. MCU 
aggregates CPU, RAM, ROM and various IO interfaces. MCU is of low price, small volume, low power consumption, but carries powerful functions. Currently the mainstream STM32 and A6, A7 produced by ARM Company all belong to the scope of MCU. Only by adding power at the outside of MCU and setting external interrupted circuit, people could easily realize the control of MCU. The minimum system construction of MCU is very simple and powerful. It could realize power data collection, inputting data management and so on. Therefore, MCU, which is already widely adopted in industry area, shall be taken as the control core of the circuit.

\section{The model selection of MCU and sensor 3.1. The model selection of MCU}

The MCU is the core of the whole control system, and central nerves of the whole control system. During the selection of MCU, processing speed, data storage capability, price and communication mode shall be mainly considered. After comprehensive consideration, this essay selects AT89C51 as main control chip of the control system. AT89C51 has the following characteristics: $4 \mathrm{~KB}$ inside flash memory and $128 \mathrm{~kb}$ program storage. The highest frequency of AT89C51 could reach $32 \mathrm{Mhz}$, with 8-digit number data handling capacity, 32 IO interfaces and two timers. The model selection of 3.2 sensor in the most popular temperate senor in the market are mostly produced by DALLAS Semiconductor Company. This essay adopts DS18B20 produced by DALLAS Semiconductor Company as the temperature sensor of the system. DS18B20 is a kind of intelligent temperature sensor widely used in industrial control. It is mainly applied in industry, military, aerospace, medical and other areas. DS18B20 converts collected temperature into binary code, storing in inside RAM, and transform to MCU or other control core by binary code [3].

\subsection{The development and application of tempera- ture measurement}

Thermocouple sensor is a kind of frequently used sensor. This kind of sensor is of super quality and competitive price, with high precision. Compared with other sensors, its overall structure is very simple, but its measurement scope is wide and of quick reaction. However, currently the signal it transforms is relatively weak, only recognizing a few millivolts to dozens of millivolt voltage. Therefore, usually during conversion, first process the signal to realize by amplified circuit. Generally, Thermocouple sensor is used to realize it, a simple access. In addition, cold compensation method could also be adopted for thermocouple sensor, that is to say, when the temperature of thermocouple sensor is not $0{ }^{\circ} \mathrm{C}$, its output potential would gradually tend to $0{ }^{\circ} \mathrm{C}$. Therefore, compensation method needs to be adopted to rectify to fix other situation to rectify, therefore guaranteeing unchanged temperature.

\subsection{The design of MCU temperature control system}

\subsubsection{The hardware of temperature control system}

The hardware circuit of circuit temperature control system mainly contains temperature sensor circuit, D/A conversion circuit, $\mathrm{A} / \mathrm{D}$ conversion circuit, $\mathrm{MCU}$ minimum system circuit, band-pass filter circuit, amplified circuit and some circuit multiplexer switch circuit and magnetic valve control circuit and so on. Of course, in order to realize different design requirements, different circuit and different configuration could still be built outside the MCU system, for example, matrix circuit could be adopted to realize keyboard control, buzzer could be adopted to realize temperature alarm, or LCD module could be adopted to display collected temperature on the panel. By these different outside modules, MCU temperature control system could be further perfected. The software development of 5.2 temperature control system and software programming of adopt $\mathrm{C}$ programming language to realize MCU control. By $\mathrm{C}$ programming language, temperature collection frequency control, temperature display and control of MCU could be realized. The procedure of control system mainly contains main program and subprogram. The main program is mainly in charge of realizing MCU initialization and initial setting of temperature sensor (reading temperature, processing temperature, storage temperature) and the initialization of keyboard and LCD display. The main program adopts cyclic query to realize temperature collection and control and temperature display. The main function of main program is to timely collect temperature and read the binary code in the sensor to $\mathrm{MCU}$, with the processing of MCU to transform into decimalism and display on the LCD.

\subsubsection{System debugging and function realization}

Proceed system debugging after the production of the whole machine, first energize thermoelectric device, the system is in stand-by mode, the output voltage of U2 (LM7805) VCC is normal $+5 \mathrm{~V}$, at that time, green power light LED1 is on, 2-digit numbering tube displays "-_-”, red LED2 (meaning heating) and blue light LED3 (meaning refrigeration) are both off. By the control button S1 starting, the whole machine begins to work; the initial quantitative power on the numbering tube is displayed as 60 , meaning the MCU is working normally. When the work starts, press S2 "power +" button to increase refrigeration/ heating power value; press S3 "power-" button to reduce refrigeration/heating power; press $\mathrm{S} 4$ to control the cyclic conversion of thermoelectric cooler's three working models as heating (red light LED2 on), pause (both red and blue light off), refrigeration (blue light LED3 on), meaning $\mathrm{H}$ bridge refrigeration / heating mode conversion circuit is normal. The thermoelectric cooler could realize refrigeration and heating function, therefore to realize the control of semiconductor thermoelectric cooler on temperature $[2,4]$. 


\section{How to realize temperature control}

4.1. Adopting pure hardware closed-loop control system Pure hardware closed-loop control system has the characteristic of quick speed, but its reliability and control precision are relatively low. In addition, pure hardware closedloop control system is inconvenient to install, the circuit is complex and it's very hard to realize the requirements in the title.

\subsection{FPGA/CPLD or adopting FPGA/CPLD with IP in- ternal core}

The key point of this mode is adopting FPGA/CPLD to realize collection, display and other functions. It has advantages of compact structure, being able to realize complex control, and easy to operate. However, the cost of FPGA/ $\mathrm{CPLD}$ is very high and the debugging is complex.

\subsection{The mode of combining $M C U$ and high precision temperature sensor}

First adopt MCU to complete control system and signal analysis, then use high-end temperature sensor to complete signal collection. Adopting the mode of combining MCU and high precision temperature sensor could effectively avoid the disadvantages of the above two modes. Therefore, this essay adopts MCU to control temperature.

\section{Temperature control system structure}

The structure of temperature control system is as shown in Figure 1 . The system adopts accumulator to supply power, after one stage direct current conversion, converting voltage to proper grade to supply power for semiconductor thermoelectric cooler, the refrigeration interface of thermoelectric cooler reduce temperature of liquid in the container by heat exchanger. The liquid temperature is sent to ADC inside of MSP430 MCU to convert to digital quantity, digital PID program calculates corresponding duty

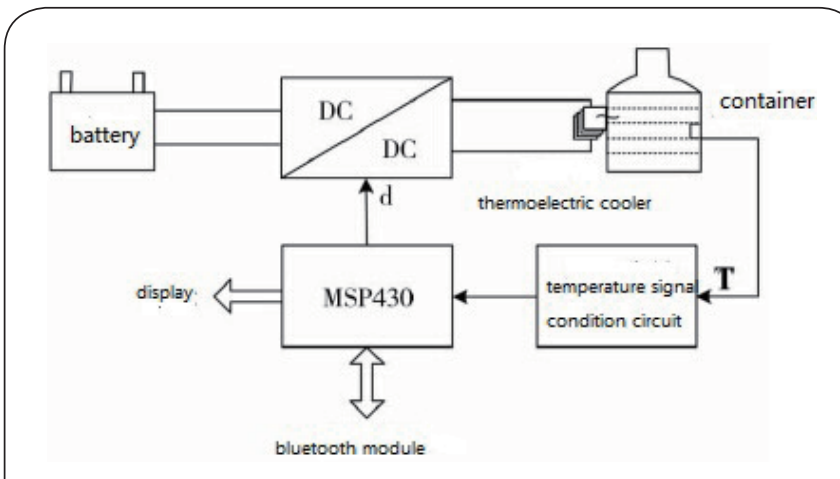

Figure 1. Temperature control system structure. ratio to control DC-DC converter, to realize the control of thermoelectric cooler. Meantime, reading and programming liquid crystal circuit through IO interface of MPS430 MCU, communicate through asynchronous serial port and Bluetooth module, receive temperature set value and send current system temperature value [5].

\section{Conclusion}

With the development of science and technology, semiconductor refrigeration technology has already been adopted in many areas; semiconductor refrigeration technology has made great contribution to the rapid development of economic construction and environment protection. MCU is the core component, taking semiconductor thermoelectric cooler as refrigeration component, developing a set of semiconductor thermoelectric cooler temperature control system based on MCU. Research indicates that this system, by controlling switch power supply's unit circuit with power dissipation, control the working status of semiconductor thermoelectric cooler (refrigeration / heating conversion, adjustable power), to reach the temperature of measuring and controlling some equipment, and guarantee the goal of proper functioning. This system is easy to operate, and it works safely and reliably, which could meet the overall performance requirements, and is of great application prospect and promotion value.

\section{Conflicts of interest}

These authors have no conflicts of interest to declare.

\section{Author's contributions}

These authors contributed equally to this work.

\section{References}

1. Yuan, M., Zhang, L. J., \& Dong, S. P. (2010). Temperature Control Experiment Platform Development on Semiconductor Thermoelectric Cooler. Experiment Technology and Management, 12, 73-76.

2. An, W. C., \& Song, X. L. (2010). Semiconductor Refrigeration Temperature Control Circuit Research on MCU. Technology Wind, 22, 245.

3. Li, H. D., Zhang, J. M., \& Wang, H. Z. (2013). Temperature Collection Control System Design on Semiconductor Thermoelectric Cooler. Tianjin University of Technology and Education Academic Journal, 01, 21-24.

4. An, W. C. (2011). Semiconductor Refrigeration Temperature Control Circuit Design on MCU. North China Electric Power University.

5. Yu, W. X., \& Dai, J. M. (2013). Temperature Control System Based on Semiconductor Refrigeration Components. Automation Technology and Application, 10, 60-64. 